\title{
PLANAR COVERINGS OF CLOSED RIEMANN SURFACES
}

\author{
JIRO TAMURA
}

To Professor Kiyoshi NoshiRo on the occasion of his 60th birthday

Several years ago, K. Oikawa, my colleague, investigated the properties of Schottky coverings of closed Riemann surfaces, leaving an interesting problem as open [3]:

Does exist a Schottky covering between the basic surface and a given planar covering?

A principal aim of this paper is to give an affirmative answer to the above problem.

In spite of the purely topological character of the problem, we must use some analytic means, namely the properties of Fuchsian group as a group of cover transformations of the universal covering of the closed surface. These are discussed in $\S 3$. On the other hand, in $\S 2$ will be treated a combinatorial topological problem. Results in both paragraphs will be used in $\S 4$ to prove the main theorem 2.

The author must pay his regard to Oikawa, the proposer of the problem, and express his warmest thanks for his friends in Universidad Central de Venezuela.

\section{$\S 1$. Introduction}

Let $W$ be a closed Riemann surface of genus $g>1$ and $F$ be the fundamental group of $W$.

We shall consider a covering $\tilde{W}$ of $W$, which is normal in the sense of Ahlfors-Sario [1], namely, unverzweigt and unbegrenzt fortsetzbar in Weyl's sense [4], and possessing a normal subgroup $G \subset F$ as its fundamental group. In this way, a normal subgroup $G$ of $F$ and a normal covering $\tilde{W}$ of $W$ correspond one-to-one; we shall represent the relation as follows:

Received June 22, 1966. 


$$
\widetilde{W}=\tilde{W}(G), \quad G=G(\tilde{W}) .
$$

If another covering $\tilde{W}_{1}$ of $W$ is at the same time a covering of $\tilde{W}, \tilde{W}_{1}$ is called stronger than $\tilde{W}$, and is denoted by

$$
\widetilde{W}_{1} \geq \tilde{W} .
$$

This relation is equivalent to

$$
G\left(\widetilde{W}_{1}\right) \subset G(\widetilde{W})
$$

The strongest covering is the universal covering, which may be considered as the upper half plane:

$$
U=\{z=x+i y ; y>0\} .
$$

We shall use the letters to represent the projections as follows:

$w=p(z)$ is the projection from $U$ onto $W$;

$\widetilde{w}=\tilde{p}(z)$ is that from $U$ onto $\tilde{W}$;

$w=\pi(\widetilde{w})$ is that from $\tilde{W}$ onto $W$.

The totality of cover transformations of $U$ w.r.t. $W$ forms a Fuchsian group $\emptyset$, isomorphic to $F$. We shall denote by $\Gamma$ the normal subgroup of $\Phi$ corresponding to $G \subset F ; \Gamma$ is the group of cover transformations of $U$ w.r.t. $\tilde{W}=\widetilde{W}(G)$.

When $\tilde{W}$ is a domain in the complex plane, $\tilde{W}$ is called a planar covering; in this case, let us call the corresponding groups $G$ and $\Gamma$ also "planar".

Let $A$ be a subset of $F$ or $\emptyset$. We shall denote the smallest normal subgroup containing $A$ by the symbol $[A]$.

A base of $F$

$$
\left\{a_{1}, b_{1}, \ldots, a_{g}, b_{g}\right\}
$$

is canonical when they have only one relation

$$
a_{1} b_{1} a_{1}^{-1} b_{1}^{-1} \cdots a_{g} b_{g} a_{g}^{-1} b_{g}^{-1}=1 \text { ( } 1 \text { is the identity). }
$$

Following Ahlfors-Sario, the Schottky covering of $W$ is defined as a covering $\tilde{W}=\tilde{W}(G)$, where

$$
G=\left[a_{1}, \ldots, a_{g}\right]
$$

for some canonical base of $F$; in this case, we shall call $G$ and $\Gamma$ also of Schottky.

A Schottky covering is planar [1], [3]. Moreover, Oikawa proved the following exactly [3]: 
THEOREM. A Schottky covering has no planar coverings which are strictly weaker than itself.

We shall prove that every planar covering is a covering of some Schottky covering.

Then we shall be able to characterize a Schottky covering as a minimal normal planar covering, free from bases.

\section{§. A theorem on Schottky Covering}

Let

$$
c_{1}, c_{2}, \ldots, c_{h}
$$

be a sequence of Jordan curves on the closed surface $W$ such that:

(A) As elements of the fundamental group $F$,

$$
c_{i} \notin\left[c_{0}=1, c_{1}, \ldots, c_{i-1}\right] \quad(i=1,2, \ldots, h) ;
$$

(B) As point sets in $W$,

$$
c_{i} \cap c_{j}=\phi \quad \text { if } i \neq j .
$$

We shall prove the following:

Lemma 1. If a set of Jordan curves $\left\{c_{i}\right\}$ satisfies the conditions $(\mathrm{A}),(\mathrm{B})$, then

$$
\left[c_{1}, \ldots, c_{h_{2}}\right]
$$

is contained in some Schottky group.

Proof. The curve $c_{1}$ may be a non-dividing cycle or a dividing cycle; then, cutting along $c_{1}$ we obtain a bordered surface $W^{1}$ in the former case, or two bordered surfaces $W^{2}$ and $W^{3}$ in the latter case.

The curve $c_{2}$ is contained in one of $W^{1}, W^{2}, W^{3}$, say $W^{i}$; cutting along $c_{2}$ we obtain a surface $W^{i 1}$ or two surfaces $W^{i 2}$ and $W^{i 3}$.

Continuing this process successively, we obtain at last a finite number of bordered surfaces

$$
W_{1}, W_{:}, \ldots, W_{s}
$$

Every curve $c_{i}$ is divided into two "banks" $c_{i}^{\prime}$ and $c_{i}^{\prime \prime}$. When $c_{i}^{\prime}$ and $c_{i}^{\prime \prime}$ belong to a same surface $W_{n}$, we rewrite

$$
c_{i}^{\prime}=e_{i}, c_{i}^{\prime \prime}=e_{i}^{-1}
$$


when $c_{i}^{\prime}$ and $c_{i}^{\prime \prime}$ belong to different surfaces $W_{m}$ and $W_{n}$ respectively, we rewrite

$$
c_{i}^{\prime}=d_{i}, c_{i}^{\prime \prime}=d_{i}^{-1} .
$$

Now suppose $W_{1}$ contains the borders

$$
e_{1}, e_{1}^{-1}, \ldots, e_{p}, e_{p}^{-1} ; d_{1}, \ldots, d_{q},
$$

in which the indices and the directions are suitably changed. Then we can get a canonical polygon $P_{1}$ of $W_{1}$ whose boundary is of the form [1]:

$$
\begin{aligned}
\partial P_{1} & =a_{1} b_{1} a_{1}^{-1} b_{1}^{-1} \cdots a_{r} b_{r} a_{r}^{-1} b_{r}^{-1} \\
& \times f_{1} e_{1} f_{1}^{-1} g_{1} e_{1}^{-1} g_{1}^{-1} \cdots f_{p} e_{p} f_{p}^{-1} g_{p} e_{p}^{-1} g_{p}^{-1} \\
& \times h_{1} d_{1} h_{1}^{-1} \cdots h_{q} d_{q} h_{q}^{-1} .
\end{aligned}
$$

For simplicity we shall write the above product as follows:

$$
\Pi a b a^{-1} b^{-1} \cdot \Pi f e f^{-1} g e^{-1} g^{-1} \cdot \Pi h d h^{-1} \text {. }
$$

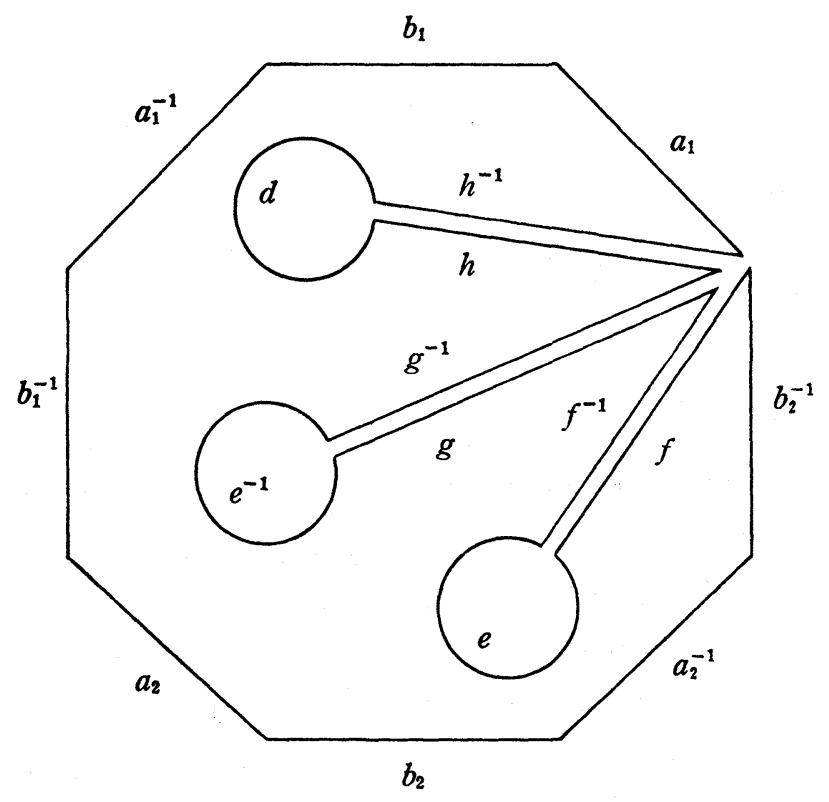

FIG. 1

We can change freely the order of the terms $f e f^{-1}, g e^{-1} g^{-1}, h d h^{-1}$, which correspond to the borders of $W_{1}$, using the elementary deformations. Also can we take the same point of $W_{1}$ as the final point of $g_{i}$ and $f_{i}$ for each $i$; namely $g_{i} f_{i}^{-1}$ is a cycle on $W_{1}$. 
Now put

$$
a_{r+i}=f_{i} e_{i} f_{i}^{-1}, \quad b_{r+i}=g_{i} f_{i}^{-1}(i=1, \ldots, p),
$$

then

$$
a_{r+i} b_{r+i} a_{r+i}^{-1} b_{r+i}^{-1}=f_{i} e_{i} f_{i}^{-1} g_{i} e_{i}^{-1} g_{i}^{-1} .
$$

Hence the boundary of $P_{1}$ can be written as follows :

$$
\partial P_{1}=\Pi a b a^{-1} b^{-1} \cdot \Pi h d h^{-1},
$$

and it is evident

$$
\left[e_{1}, \ldots, e_{p}, d_{1}, \ldots, d_{q}\right] \subset\left[a_{1}, \ldots, a_{r+p}, d_{1}, \ldots, d_{q}\right] .
$$

In the same way, every $W_{n}$ can be represented by the canonical polygon $P_{n}$ whose boundary is similar as $\partial P_{1}$.

Now we suppose

$$
d_{1} \subset \partial P_{1}, d_{1}^{-1} \subset \partial P_{2}
$$

Let us denote

$$
\partial P_{2}=\prod_{i=1}^{s} a_{i}^{\prime} b_{i}^{\prime} a_{i}^{\prime-1} b_{i}^{\prime-1} \cdot \prod_{j=1}^{t} h_{j}^{\prime} d_{j}^{\prime} h_{j}^{\prime-1}
$$

and suppose that $d_{1}^{-1}$ is the last border $d_{t}^{\prime}$ without loss of generality. Identifying $d_{1}$ on $\partial P_{1}$ and $d_{t}^{\prime}$ on $\partial P_{2}$, we obtain a new polygon $P_{1}+P_{2}$, whose boundary is of the form

$$
\begin{aligned}
\partial\left(P_{1}+P_{2}\right) & =\Pi a b a^{-1} b^{-1} \cdot h_{1} h_{t}^{\prime-1} \cdot \Pi a^{\prime} b^{\prime} a^{\prime^{-1}} b^{1^{-1}} \\
& \times \Pi h^{\prime} d^{\prime} h^{\prime-1} \cdot h_{t}^{\prime} h_{1}^{-1} \cdot \Pi h d h^{-1} .
\end{aligned}
$$

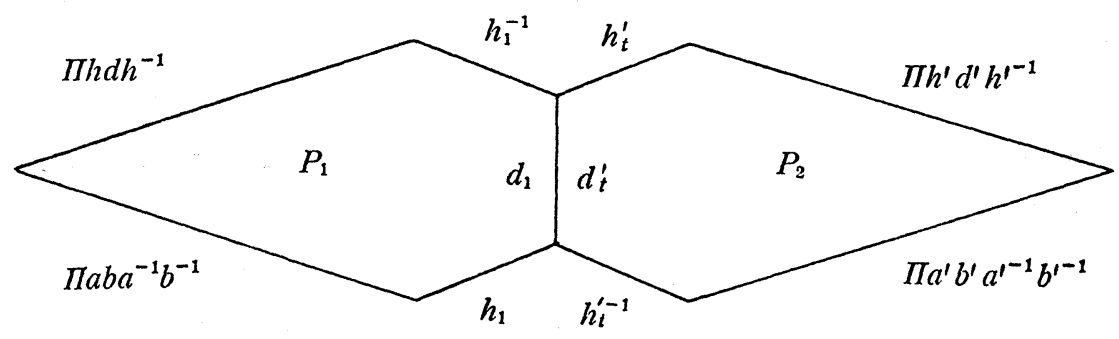

FIG. 2

We shall transform $a, b, h$, and $d$ by $h_{1}^{-1}$ and write

$$
\bar{a}=h_{1}^{-1} a h_{1}, \bar{b}=h_{1}^{-1} b h_{1}, \bar{h}=h_{1}^{-1} h h_{1}, \bar{d}=h_{1}^{-1} d h_{1} .
$$

In the similar way, let us transform $a^{\prime}, b^{\prime}, h^{\prime}, d^{\prime}$ by $h_{t}^{\prime}$. Then we obtain the 
following form (with suitable change of notations):

$$
\partial\left(P_{1}+P_{2}\right)=\Pi a b a^{-1} b^{-1} \cdot \Pi h d h^{-1},
$$

in which the common border $d_{1}$ is not contained. However, as easily seen, $d_{1}$ can be generated by $a$ and $d$ contained in $\partial\left(P_{1}+P_{2}\right)$. Hence

$$
\left[e, d \subset \partial P_{1} ; e, d \subset \partial P_{2}\right] \subset\left[a, d \subset \partial\left(P_{1}+P_{2}\right)\right]
$$

Continuing this process successively, we can get at last a polygon

$$
P=P_{1}+P_{2}+\cdots+P_{N}
$$

whose boundary is of the form

$$
P=\Pi a b a^{-1} b^{-1} \text {. }
$$

Hence, $P$ is nothing but a canonical polygon of $W$ itself. Moreover we can verify inductively

$$
\left[c_{1}, \ldots, c_{h}\right] \subset[a ; a \subset \partial P]
$$

the right side of which is a Schottky covering group.

q.e.d.

Let us ncis tirai the condition (A) is not used in the above proof. However, under this co: litinn we can see that every bordered surface $W_{n}$ (pasted along $e_{i}$ ) has a positive genus $g_{n}$; In fact, if the boundary $\partial P_{n}$ contains no terms of the form $a b a^{-1} b^{-1}$, then we can obtain a relation between $\left\{c_{i}\right\}$, which contradicts (A).

Since each if $\{e\}$ and $\{d\}$ does not exceed $g_{1}+\cdots+g_{N}=g$, we conclude

$$
h \leq 2 g \text {. }
$$

Consequently, there is no infinite sequence $\left\{c_{n}\right\}$ of Jordan curves satisfying (A) and (B).

Hence, when the infinite sequence $\left\{c_{n}\right\}$ satisfies $(B)$ only, we can select a suitable finite subsequence $\left\{d_{i}\right\}_{i=1}^{h}$ which satisfies (A), (B) and

$$
\left[d_{1}, \ldots, d_{h}\right]=\left[\left\{c_{n}\right\}\right] .
$$

Then we have the following:

THEOREM 1. Let $\left\{c_{n}\right\}$ be a sequence of Jordan curves in the closed surface $W$ such that 


$$
c_{i} \cap c_{j}=\phi \quad(i \neq j)
$$

Then there exists a Schottky group containing $\left[\left\{c_{n}\right\}\right]$.

\section{§. Fuchsian groups of closed Riemann surfaces}

Let $U$ be the upper half plane

$$
\{z=x+i y ; y>0\}
$$

and $(S$ be the group of all linear transformations which leave $U$ invariant.

We shall introduce in $U$ the Poincaré metric

$$
d s=\frac{|d z|}{y}
$$

which defines a non-Euclidean geometry in $U$; we use the words of elementary geometry in the sense of non-Euclid: for example, a "straight line" $l$ is a inner arc of a circle orthogonal to $\partial U$. Let us denote a "directed segment" from $z_{1}$ to $z_{2}$ by $s\left(z_{1}, z_{2}\right)$, and the "distanse" between $z_{1}$ and $z_{2}$ by $\rho\left(z_{1}, z_{2}\right)$.

$B$ is the group of "motions" of plane $U$, leaving the distance $\rho$ invariant.

Definition 1. Let $\varphi$ be an arbitrary transformation of 8 . We shall define the norm $\|\varphi\|$ of $\varphi$ as follows:

$$
\|\varphi\|=\inf _{z \in U} \rho(z, \varphi(z)) .
$$

We can prove easily some properties of "norm" as follows :

Proposition 1.

$$
\left\|\sigma \sigma^{-1}\right\|=\|\varphi\|
$$

for all $\varphi, \sigma \in \mathbb{S}$, namely the norm $\|\varphi\|$ is invariant by the inner transformation.

Proof. $\left\|\sigma \varphi \sigma^{-1}\right\|=\inf \rho\left(z, \sigma \varphi \rho^{-1}(z)\right)=\inf \rho\left(\sigma^{-1}(z), \varphi_{\sigma}^{-1}(z)\right)=\inf \rho(z, \varphi(z))=\|\varphi\|$.

Proposition 2. (i) If $\varphi \in \mathbb{B}$ is elliptic or parabolic, $\|\varphi\|=0$. (ii) If $\varphi \in \mathbb{B}$ is hyperbolic,

$$
\|\varphi\|=|\log K|
$$

where $K$ is the multiplier of $\varphi^{11}$.

1) See, for example, Ford [2]. 
Proof. (i) If $\varphi$ is elliptic, one of the fix points $a$ is in $U$. Hence

$$
\|\varphi\|=\rho(a, \varphi(a))=0 .
$$

If $\varphi$ is parabolic we can consider without loss of generality, the only fix point of $\varphi$ is $\infty$ (Prop. 1). Then

$$
\varphi(z)=z+c(c \in \mathbf{R}) .
$$

Uniting $z_{0}=y_{0}$ and $\varphi\left(z_{0}\right)$ by a Euclidean segment $C$, we get

$$
\|\varphi\| \leq \rho\left(y_{0}, y_{0}+c\right) \leq \int_{c} \frac{|d x|}{y_{0}}=\frac{|c|}{y_{0}} .
$$

Let $y_{0} \rightarrow+\infty$, then we obtain $\|\varphi\|=0$.

(ii) Let $\varphi$ be hyperbolic. In this case two fix points of $\varphi$ are on $x$-axis, which may be supposed 0 and $\infty$ without loss of generality. Hence

$$
\varphi(z)=K z,
$$

where $K$ is the multiplier of $\varphi(K>0, K \neq 1)$. Putting

$$
z=x+i y=r e^{i \theta},
$$

we shall estimate Poincaré metric:

$$
d s=\frac{|d z|}{y}=\frac{\sqrt{d r^{2}+r^{2} d \theta^{2}}}{r \sin \theta} \geq \frac{|d r|}{r} .
$$

Suppose $C$ is a segment from $z$ to $K z$,

$$
\rho(z, \varphi(z))=\int_{c}\left|\frac{d z \mid}{y}\right| \geq\left|\int_{r}^{K r} \frac{d r}{r}\right|=|\log K|,
$$

where the equality holds if and only if $d \theta=0, \sin \theta=0$; namely, $z$ is on the imaginary axis.

Definition 2. Let $\varphi \in \mathbb{B}$ be hyperbolic. The "straight line" determined by two fix points of $\varphi$ is called the axis of $\varphi$.

By the proof of prop. 2, the followings are evident:

PROPOSITION 3. For any hyperbolic transformation $\varphi \in \mathbb{B}$,

$$
\|\varphi\|=\rho(z, \varphi(z))
$$

if and only if $z$ belongs to the axis of $\varphi$. 
Proposition 4. Let $\varphi$ be a hyperbolic transformation with axis $l$. Then $\sigma_{\sigma}{ }^{-1}$ is also hyperbolic whose axis is $\sigma(l)$.

Now let us return to the closed Riemann surface $W$ and its universal covering $U$.

The group $\Phi$ of cover transformations of $U$ w.r.t. $W$ is a special sort of Fuchsian groups, which is characterized by the following conditions:

$\left.1^{\circ}\right)$ is free from elliptic transformations;

$\left.2^{\circ}\right) \Phi$ has a compact fundamental region $\Delta^{2}$.

We shall call $\Phi$ the Fuchsian group of $W$, for brevity.

Proposition 5. For the Fuchsian group $\emptyset$ of a given closed surface $W$, exists a positive number $r$ such that

$$
z_{1} \neq z_{2}, \rho\left(z_{1}, z_{2}\right)<r \text { implies } z_{1} \neq z_{2}(\emptyset) .
$$

The proof is simple by the compactness of the fundamental region $\Delta$.

Proposition 6. Every transformation of $\Phi$ is hyperbolic except $\varphi=1$ (identity), and the set of norms

$$
\{\|\varphi\| ; \varphi \in \emptyset\}
$$

is discrete in $\mathbf{R}$.

Proof. For any $\varphi \neq 1$ of $\emptyset$

$$
\|\varphi\| \geq r>0
$$

by Prop. 5 . Hence, $\Phi$ has no parabolic transformations.

If we take a point $z$ on the axis of $\varphi$,

$$
\|\varphi\|=\rho(z, \varphi(z)) .
$$

Then exists a suitable $\sigma \in \mathscr{D}$ such that $\sigma(z)=z_{0}$ belongs to the fixed fundamental region 4 . Put

$$
\varphi_{0}=\sigma \varphi_{\sigma}^{-1}
$$

and $z_{0}$ is a point on the axis of $\varphi_{0}$. Let us denote by $\emptyset_{0}$ the collection of all $\varphi_{0}$ whose axis pass through 4 . Then

2) Some authors define the fundamental region as a open one. However we consider it as a closed region for convenience. 


$$
\{\|\varphi\| ; \varphi \in \Phi\}=\left\{\left\|\varphi_{0}\right\| ; \varphi_{0} \in \Phi_{0}\right\} .
$$

- On the other hand, for any positive number $R$, the compact set

$$
\{z ; \rho(z, \Delta) \leq R\}
$$

is covered by a finite number of congruent figures $\varphi(\Delta)$. Hence the number of real values $\left\|\varphi_{0}\right\| \leq R$ is finite.

Let $l_{1}, l_{2}$ be axis of $\varphi_{1}, \varphi_{2} \in \emptyset$ respectively; there may be three cases as follows :

$\left.1^{\circ}\right) l_{1} \cap l_{2}=\phi$

$\left.2^{\circ}\right) l_{1} \cap l_{2}=\left\{z_{0}\right\}$;

$\left.3^{\circ}\right) l_{1}=l_{2}$.

The last case occurs if and only if $\varphi_{1}$ and $\varphi_{2}$ have common fix points. Hence we can verify easily:

Proposirion 7. Let $I$ be a subgroup of $\emptyset$. The totality of $\varphi \in \Gamma$, possessing a given fixed axis $l$, forms a free cyclic group generated by a suitable $\varphi_{0} \in \Gamma$.

\section{$\S$ 4. Planar coverings of closed Riemann surfaces}

Let us use the same notations as in former paragraphs, and suppose $\widetilde{W}$ is a normal planar covering of a closed surface $W . U$ is also a universal covering of $\tilde{W}$ and the group $I$ of cover transformations of $U$ w.r.t. $\tilde{W}$ is a normal subgroup of $\Phi ; I$ is isomorphic to the fundamental group $G$ of $\widetilde{W}$.

Let $C$ be any curve (closed or not) in $U$, whose terminal points are $z_{0}, z_{1}$. Consider the projection of $C$ on $W$ and $\tilde{W}$ :

$$
\begin{aligned}
& c=p(C) \text { on } W ; \\
& \widetilde{c}=\tilde{p}(C) \text { on } \tilde{W} .
\end{aligned}
$$

Evidently, $c$ is closed if and only if

$$
z_{0} \equiv z_{1} \quad(\emptyset)
$$

$\tilde{c}$ is closed if and only if

$$
z_{0} \equiv z_{1} \quad(\Gamma) .
$$

Now let us put $\gamma_{0}=1 \in \Gamma$ and take a transformation $\gamma_{1}$ which has the minimum norm in $\Gamma-\left[\gamma_{0}\right]$. If $\left[\gamma_{0}, \gamma_{1}\right] \neq \Gamma$, we shall take $\gamma_{2}$ which has the minimum norm in $\Gamma-\left[\gamma_{0}, \gamma_{1}\right]$. 
Continuing such processes, we obtain a finite or infinite sequence of transformations

$$
\gamma_{1}, \gamma_{2}, \ldots, \gamma_{n}, \ldots
$$

Let us denote the totality of indices of $\gamma_{n}$ by $N=\{1,2, \ldots, n, \ldots\}$. Using Prop. 6 it is easily seen that $\gamma_{n}$ has the following properties:

(I) $\left[r_{n} ; n \in N\right]=\Gamma$.

(II) If $\varphi \in \Gamma$, $\|\varphi\|<\left\|\gamma_{n}\right\|$, then

$$
\varphi \in\left[\gamma_{1}, \gamma_{2}, \ldots, \gamma_{n-1}\right] .
$$

We shall take a point $z$ on the axis $l_{n}$ of $\gamma_{n}$, and set

$$
\begin{aligned}
& C_{n}=s\left(z, \gamma_{n}(z)\right), z \in l_{n} ; \\
& c_{n}=p\left(C_{n}\right) ; \\
& \widetilde{c}_{n}=\tilde{p}\left(C_{n}\right) .
\end{aligned}
$$

Let us remark that every $c_{n}$ is invariant not only as a homotopy class of $F$ but also as a point set in $W$, when the inicial point $z \in l_{n}$ is changed; the same is true for $\tilde{c}_{n}$.

We shall prove first

(III) $\tilde{c}_{n}$ is a Jordan curve in $\widetilde{W}$.

Proof. If $\widetilde{c}_{n}$ is not of Jordan in $\tilde{W}, \widetilde{c}_{n}$ can be divided into two closed curves $\widetilde{c}$ and $\tilde{c}^{\prime}$ on $\tilde{W}$. Hence there exist three points $z_{1}, z_{2}, z_{3}$ on the axis $l_{n}$ of $\gamma_{n}$ in this order, such that

$$
\begin{aligned}
& \widetilde{p}\left(s\left(z_{1}, z_{2}\right)\right)=\tilde{c}, \\
& \widetilde{p}\left(s\left(z_{2}, z_{3}\right)\right)=\bar{c}^{\prime} .
\end{aligned}
$$

Namely there exist two transformations $\varphi$ and $\psi$ in $\Gamma$, such that

$$
\varphi\left(z_{1}\right)=z_{2}, \psi\left(z_{2}\right)=z_{3}
$$

and

$$
\begin{aligned}
& \|\varphi\| \leq \rho\left(z_{1}, z_{2}\right)<\rho\left(z_{1}, z_{3}\right)=\left\|\gamma_{n}\right\| \\
& \|\varphi\| \leq \rho\left(z_{2}, z_{3}\right)<\rho\left(z_{1}, z_{3}\right)=\left\|\gamma_{n}\right\| .
\end{aligned}
$$

According to (II)

$$
\varphi, \psi \in\left[r_{0}, \ldots, r_{n-1}\right] .
$$

hence 


$$
\gamma_{n}=\psi \varphi \in\left[\gamma_{0}, \ldots, \gamma_{n-1}\right]
$$

which is a contradiction.

q.e.d.

(IV) Every curve $\widetilde{c}$ in $\widetilde{W}$ whose projection is $\pi(\tilde{c})=c_{n}$, is of Jordan in $\widetilde{W}$.

The proof is immediate from (III) and the normality of the covering $\tilde{W}$.

(V) Let $l_{m}, l_{n}$ be the axis of $\gamma_{m}, \gamma_{n}$ respectively, and $l=\sigma\left(l_{m}\right), l^{\prime}=\tau\left(l_{n}\right)$, where $\sigma$, $\tau \in \emptyset$. Then $l \cap l^{\prime}=\phi$ or $l=l^{\prime}{ }^{3 \prime}$

Proof. $l$ and $l^{\prime}$ are the axis of $\gamma=\sigma \gamma_{m} \sigma^{-1}$ and $\gamma^{\prime}=\tau \gamma_{n} \tau^{-1}$ respectively (Prop. 4), and $\left\|r_{1}=\right\| r_{m}\|,\| r^{\prime}\|=\| r_{n} \|$.

Suppose that $l$ and $l^{\prime}$ intersect at $z_{0}$.

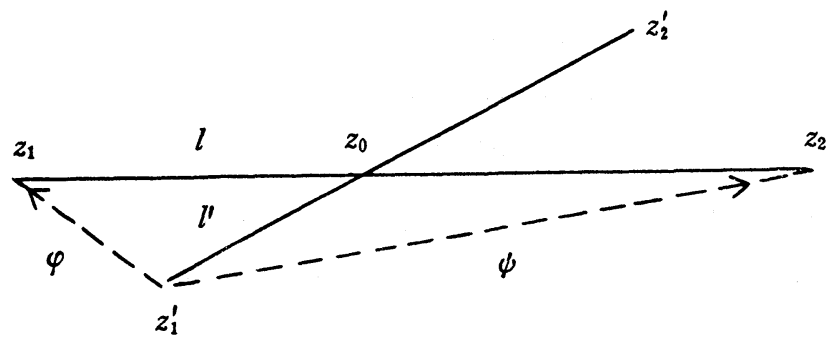

FIG. 3

Let us take (temporarily) arbitrary inicial points $z_{1} \in l, z_{1}^{\prime} \in l^{\prime}$ and put $z_{2}=r\left(z_{1}\right), z_{2}^{\prime}=\gamma^{\prime}\left(z_{1}^{\prime}\right)$. Since

$$
\widetilde{c}=\tilde{p}\left(s\left(z_{1}, z_{2}\right)\right), \tilde{c}^{\prime}=\tilde{p}\left(s\left(z_{1}^{\prime}, z_{2}^{\prime}\right)\right)
$$

are both Jordan curves on $\bar{W}$ (IV), $\tilde{c}$ and $\tilde{c}^{\prime}$ must have another common point $\widetilde{w}_{1}$ than $\widetilde{w}_{0}=\tilde{p}\left(z_{0}\right)$, because of the planar character of $\tilde{W}$. Then we can select $z_{1}, z_{1}^{\prime}$ as inicial points such that

$$
\begin{aligned}
& \widetilde{p}\left(z_{1}\right)=\tilde{p}\left(z_{2}\right)=\tilde{p}\left(z_{1}^{\prime}\right)=\tilde{p}\left(z_{2}^{\prime}\right)=\widetilde{w}_{1}, \\
& z_{0} \in s\left(z_{1}, z_{2}\right) \cap s\left(z_{1}^{\prime}, z_{2}^{\prime}\right) .
\end{aligned}
$$

It is clear that $z_{0}$ does not coincide with these terminal points $z_{1}, z_{2}, z_{1}^{\prime}, z_{2}^{\prime}$, since $\widetilde{v}_{0} \neq \widetilde{w}_{1}$. Without loss of generality, we can suppose

$$
\begin{aligned}
& \rho\left(z_{1}, z_{0}\right) \leq \rho\left(z_{0}, z_{2}\right), \\
& \rho\left(z_{1}^{\prime}, z_{0}\right) \leq \rho\left(z_{0}, z_{2}^{\prime}\right),
\end{aligned}
$$

3) If $l=l^{\prime}, m=n$ by Prop. 7 . 
and

$$
\rho\left(z_{1}^{\prime}, z_{0}\right) \leq \rho\left(z_{1}, z_{0}\right) \leq \rho\left(z_{0}, z_{2}\right) .
$$

Then using the elementary geometry of non-Euclid, we get

$$
\begin{aligned}
\rho\left(z_{1}^{\prime}, z_{1}\right) & <\rho\left(z_{1}^{\prime}, z_{0}\right)+\rho\left(z_{0}, z_{1}\right) \\
& \leq \rho\left(z_{0}, z_{2}\right)+\rho\left(z_{0}, z_{1}\right) \\
& =\rho\left(z_{1}, z_{2}\right) .
\end{aligned}
$$

Hence there exists a transformation $\varphi \in \Gamma$ such that

$$
\begin{aligned}
& \varphi\left(z_{1}^{\prime}\right)=z_{1}, \\
& \|\varphi\|_{\leq \rho}\left(z_{1}^{\prime}, z_{1}\right)<\rho\left(z_{1}, z_{2}\right)=\|r\|=\left\|r_{m}\right\| .
\end{aligned}
$$

By the similar way we get a $\psi \in \Gamma$ such that

$$
\psi\left(z_{1}^{\prime}\right)=z_{2},\|\psi\|<\left\|_{m}\right\| .
$$

Using (II), we conclude

$$
\varphi, \psi \in\left[\gamma_{0}, \ldots, \gamma_{m-1}\right]
$$

which implies

$$
r_{m}=\sigma^{-1} \gamma_{\sigma}=\sigma^{-1}\left(\psi \varphi^{-1}\right) \sigma \in\left[\gamma_{0}, \ldots, \gamma_{m-1}\right],
$$

which is a contradiction.

q.e.d.

The projection $c_{n}$ of $C_{n}$ on $W$ is not necessarily of Jordan. However, in the subgroup of $\Phi$ consisting of all the transformations with axis $l_{n}$, there exists a generator $\delta_{n}$ (Prop. 7). Let

$$
z \in l_{n}, D_{n}=s\left(z, \delta_{n}(z)\right), d_{n}=p\left(D_{n}\right) .
$$

It is evident that

$$
c_{n}=d_{n}^{k_{n}} \quad \text { for suitable } k_{n} \text {. }
$$

(VI) $d_{n}$ is a Jordan curve in $W$.

Proof. Suppose that $d_{n}$ is not of Jordan in $W$, then by the same discussion as in the proof of (III), we can find three points $z_{1}, z_{2}, z_{3}$ on $l_{n}$ in this order, such that

$$
\varphi\left(z_{1}\right)=z_{2}, \psi\left(z_{2}\right)=z_{3}, \delta_{n}=\psi \varphi
$$

for suitable $\varphi, \phi \in \emptyset$. Put 


$$
l=l_{n}, l^{\prime}=\varphi\left(l_{n}\right),
$$

then $l^{\prime}$ is the axis of $\delta^{\prime}=\varphi \delta_{n} \varphi^{-1}$ (Prop. 4). If $l^{\prime}=l$, namely $\varphi\left(l_{n}\right)=l_{n}, \varphi$ has the common fix points with $\delta_{n}$, which means that the axis of $\varphi$ is $l_{n}$; however, this is impossible since

$$
\|\varphi\| \leq \rho\left(z_{1}, z_{2}\right)<\left\|\delta_{n}\right\|
$$

Hence $l^{\prime}$ intersects $l$ at $z_{2}$, which contradicts (V).

q.e.d.

Thus we obtain the sequence of Jordan curves

$$
\left\{d_{n} ; n \in N\right\},
$$

each $d_{n}$ of which is the projection of $l_{n}$ as a point set in $W$, and generates $c_{n}$ as a homotopy class of $F$.

Moreover,

$$
d_{m} \cap d_{n}=\phi \text { if } m \neq n,{ }^{4}
$$

namely, $\left\{d_{n} ; n \in N\right\}$ satisfies the condition $(B)$ in $\S 2$.

Therefore, by Theorem 1, we get

THEOREM 2. If $\widetilde{W}$ is a normal planar covering of a closed Riemann surface $W$, there exists a Schottky covering $S$ such that

$$
\widetilde{W} \geq S>W .
$$

\section{$\S 5$. Additional remarks}

Theorem 2 is applicable not only to the closed Riemann surface, but to every orientable finite surface, because the latter is always homeomorphic to some of the formers.

Moreover, we shall remark that the normality of the covering is essencial; we can make an example $\tilde{W}$ such that

(i) $\tilde{W}$ is a regular planar covering in the sense of Ahlfors-Sario;

(ii) there are no Schottky coverings $S \leq \tilde{W}$.

However, we shall not treat the problem here.

1) See the footnote 3 ). 


\section{ReFerEnCES}

[1] Ahlfors-Sario, Riemann Surfaces, Princeton, 1960.

[2] Ford, Automorphic Functions, 2nd ed., New York, 1951.

[3] Oikawa, On Schottky Coverings, Shūgakuin Symposium of Function Theory, 1961. (in Japanese)

[4] Weyl, Die Idee der Riemannschen Flächen, Leipzig, 1913.

University of Tokyo

Universidad Central de Venezuela 